\title{
A machine learning approach to the automatic evaluation of machine translation
}

\author{
Simon Corston-Oliver, Michael Gamon and Chris Brockett \\ Microsoft Research \\ One Microsoft Way \\ Redmond WA 98052, USA \\ \{simonco, mgamon, chrisbkt\} amicrosoft.com
}

\begin{abstract}
We present a machine learning approach to evaluating the wellformedness of output of a machine translation system, using classifiers that learn to distinguish human reference translations from machine translations. This approach can be used to evaluate an MT system, tracking improvements over time; to aid in the kind of failure analysis that can help guide system development; and to select among alternative output strings. The method presented is fully automated and independent of source language, target language and domain.
\end{abstract}

\section{Introduction}

Human evaluation of machine translation (MT) output is an expensive process, often prohibitively so when evaluations must be performed quickly and frequently in order to measure progress. This paper describes an approach to automated evaluation designed to facilitate the identification of areas for investigation and improvement. It focuses on evaluating the wellformedness of output and does not address issues of evaluating content transfer.

Researchers are now applying automated evaluation in MT and natural language generation tasks, both as system-internal goodness metrics and for the assessment of output. Langkilde and Knight (1998), for example, employ n-gram metrics to select among candidate outputs in natural language generation, while Ringger et al. (2001) use ngram perplexity to compare the output of MT systems. Su et al. (1992), Alshawi et al. (1998) and Bangalore et al. (2000) employ string edit distance between reference and output sentences to gauge output quality for MT and generation. To be useful to researchers, however, assessment must provide linguistic information that can guide in identifying areas where work is required. (See Nyberg et al., 1994 for useful discussion of this issue.)

The better the MT system, the more its output will resemble human-generated text. Indeed, MT might be considered a solved problem should it ever become impossible to distinguish automated output from human translation. We have observed that in general humans can easily and reliably categorize a sentence as either machine- or human-generated. Moreover, they can usually justify their decision. This observation suggests that evaluation of the wellformedness of output sentences can be treated as a classification problem: given a sentence, how accurately can we predict whether it has been translated by machine? In this paper we cast the problem of MT evaluation as a machine learning classification task that targets both linguistic features and more abstract features such as ngram perplexity. 


\section{Data}

Our corpus consists of 350,000 aligned SpanishEnglish sentence pairs taken from published computer software manuals and online help documents. We extracted 200,000 English sentences for building language models to evaluate per-sentence perplexity. From the remainder of the corpus, we extracted 100,000 aligned sentence pairs. The Spanish sentences in this latter sample were then translated by the Microsoft machine translation system, which was trained on documents from this domain (Richardson et al., 2001). This yielded a set of 200,000 English sentences, one half of which were English reference sentences, and the other half of which were MT output. (The Spanish sentences were not used in building or evaluating the classifiers). We split the 200,000 English sentences $90 / 10$, to yield 180,000 sentences for training classifiers and 20,000 sentences that we used as held-out test data. Training and test data were evenly divided between reference English sentences and Spanish-to-English translations.

\section{Features}

The selection of features used in our classification task was motivated by failure analysis of system output. We were particularly interested in those linguistic features that could aid in qualitative analysis, as we discuss in section 5. For each sentence we automatically extracted 46 features by performing a syntactic parse using the Microsoft NLPWin natural language processing system (Heidorn, 2000) and language modeling tools. The features extracted fall into two broad categories:

(i) Perplexity measures were extracted using the CMU-Cambridge Statistical Language Modeling Toolkit (Clarkson and Rosenfeld, 1997). We calculated two sets of values: lexicalized trigram perplexity, with values discretized into deciles and part of speech (POS) trigram perplexity. For the latter we used the following sixteen POS tags: adjective, adverb, auxiliary, punctuation, complementizer, coordinating conjunction, subordinating conjunction, determiner, interjection, noun, possessor, preposition, pronoun, quantifier, verb, and other. (ii) Linguistic features fell into several subcategories: branching properties of the parse; function word density, constituent length, and other miscellaneous features

We employed a selection of features to provide a detailed assessment of the branching properties of the parse tree. The linguistic motivation behind this was twofold. First, it had become apparent from failure analysis that MT system output tended to favor right-branching structures over noun compounding. Second, we hypothesized that translation from languages whose branching properties are radically different from English (e.g. Japanese, or a verbsecond language like German) might pollute the English output with non-English characteristics. For this reason, assessment of branching properties is a good candidate for a languagepair independent measure. The branching features we employed are given below. Indices are scalar counts; other measures are normalized for sentence length.

$>$ number of right-branching nodes across all constituent types

$>$ number of right-branching nodes for NPs only

$>$ number of left-branching nodes across all constituent types

$>$ number of left-branching nodes for NPs only

$>$ number of premodifiers across all constituent types

$>$ number of premodifiers within NPs only

$>$ number of postmodifiers across all constituent types

$>$ number of postmodifiers within NPs only

$>$ branching index across all constituent types, i.e. the number of right-branching nodes minus number of left-branching nodes

$>$ branching index for NPs only

$>$ branching weight index: number of tokens covered by right-branching nodes minus number of tokens covered by left-branching nodes across all categories

branching weight index for NPs only

$>$ modification index, i.e. the number of premodifiers minus the number of postmodifiers across all categories 
$>$ modification index for NPs only

$>$ modification weight index: length in tokens of all premodifiers minus length in tokens of all postmodifiers across all categories

$>$ modification weight index for NPs only

$>$ coordination balance, i.e. the maximal length difference in coordinated constituents

We considered the density of function words, i.e. the ratio of function words to content words, because of observed problems in WinMT output. Pronouns received special attention because of frequent problems detected in failure analysis. The density features are:

$>$ overall function word density

$>$ density of determiners/quantifiers

$>$ density of pronouns

$>$ density of prepositions

$>$ density of punctuation marks, specifically commas and semicolons

$>$ density of auxiliary verbs

$>$ density of conjunctions

$>$ density of different pronoun types: Wh, 1st, 2nd, and 3rd person pronouns

We also measured the following constituent sizes:

$>$ maximal and average NP length

$>$ maximal and average AJP length

$>$ maximal and average PP length

$>$ maximal and average AVP length

$>$ sentence length

On a lexical level, the presence of out of vocabulary $(\mathrm{OOV})$ words is frequently caused by the direct transfer of source language words for which no translation could be found. The top-level syntactic template, i.e. the labels of the immediate children of the root node of a sentence, was also used, as was subject-verb disagreement. The final five features are:

$>$ number of OOV words

$>$ the presence of a word containing a nonEnglish letter, i.e. an extended ASCII character. This is a special case of the OOV problem.

$>$ label of the root node of the sentence (declarative, imperative, question, NP, or "FITTED" for non-spanning parses) sentence template, i.e. the labels of the immediate children of the root node.

$>$ subject-verb disagreement

\section{Decision Trees}

We used a set of automated tools to construct decision trees (Chickering et al., 1997) based on the features extracted from the reference and MT sentences. To avoid overfitting, we specified that nodes in the decision tree should not be split if they accounted for fewer than fifty cases. In the discussion below we distinguish the perplexity features from the linguistic features.

\subsection{Decision trees built using all training data}

Table 1 gives the accuracy of the decision trees, when trained on all 180,000 training sentences and evaluated against the 20,000 held-out test sentences. Since the training data and test data contain an even split between reference human translations and machine translations, the baseline for comparison is $50.00 \%$. As Table 1 shows, the decision trees dramatically outperform this baseline. Using only perplexity features or only linguistic features yields accuracy substantially above this baseline. Combining the two sets of features yields the highest accuracy, $82.89 \%$.

\begin{tabular}{|l|l|}
\hline Features used & Accuracy (\%) \\
\hline All features & 82.89 \\
Perplexity features only & 74.73 \\
Linguistic features only & 76.51 \\
\hline
\end{tabular}

Table 1 Accuracy of the decision trees

Notably, most of the annotated features were selected by the decision tree tools. Two features were found not to be predictive. The first non-selected feature is the presence of a word containing an extended ASCII character, suggesting that general OOV features were sufficient and subsume the effect of this narrower feature. Secondly, subject-verb disagreement was also not predictive, validating the consistent enforcement of agreement constraints in the natural language generation component of the MT system. In addition, only eight of approximately 5,200 observed sentence templates turned out to be discriminatory. 
For a different use of perplexity in classification, see Ringger et al. (2001) who compare the perplexity of a sentence using a language model built solely from reference translations to the perplexity using a language model built solely from machine translations. The output of such a classifier could be used as an input feature in building decision trees.

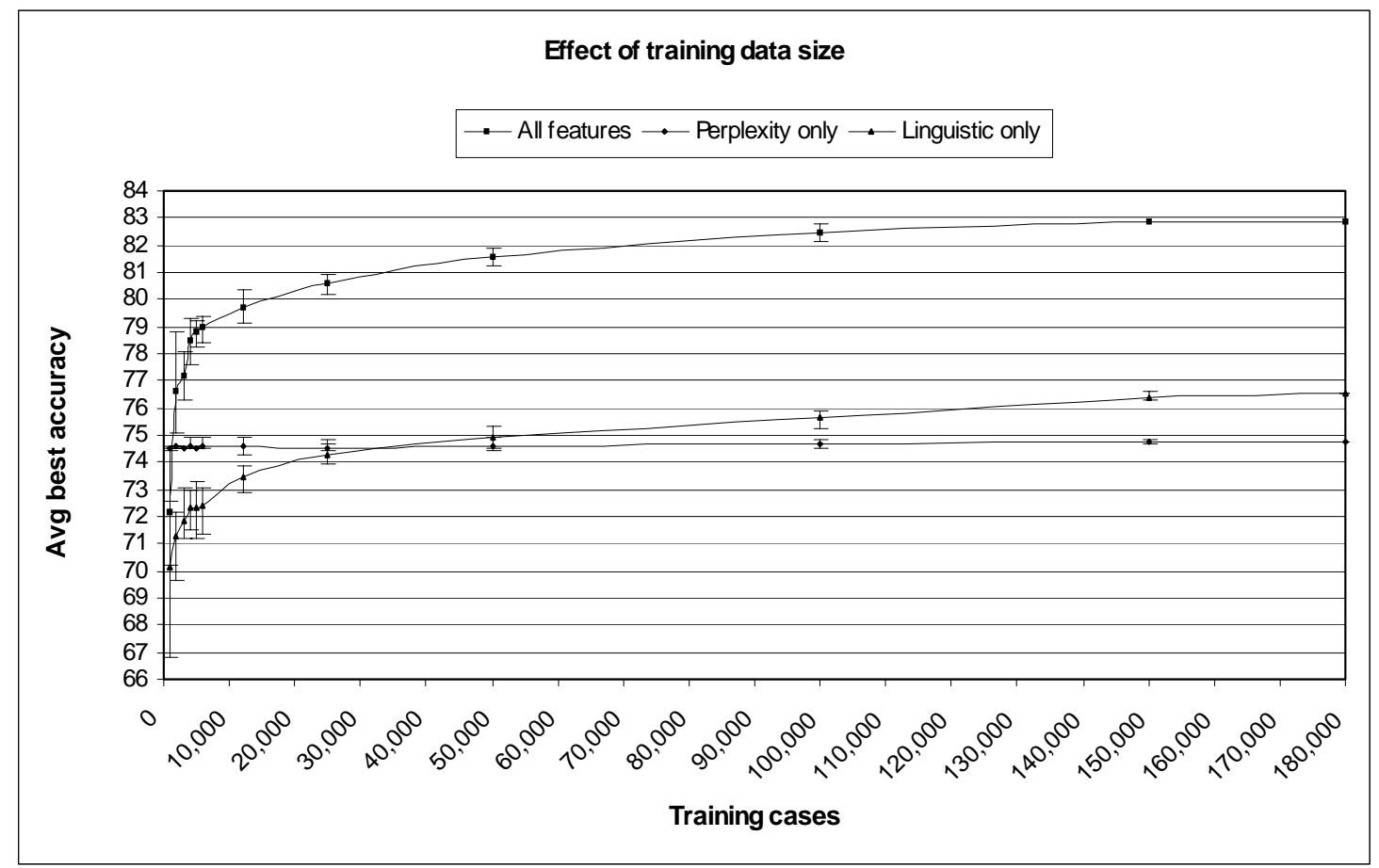

Figure 1 Accuracy with varying amounts of training data

\subsection{Varying the amount of training data}

For our experiments, we had access to several hundred thousand sentences from the target domain. To measure the effect of reducing the size of the training data set on the accuracy of the classifier, we built classifiers using samples of the training data and evaluating against the same held-out sample of 20,000 sentences. We randomly extracted ten samples containing the following numbers of sentences: $\{1,000,2,000$, $3,000,4,000,5,000,6,000,12,000,25,000$, $50,000,100,000,150,000\}$. Figure 1 shows the effect of varying the size of the training data. The data point graphed is the average accuracy over the ten samples at a given sample size, with error bars showing the range from the least accurate decision tree at that sample size to the most accurate.

As Figure 1 shows, the models built using only perplexity features do not benefit from additional training data. The models built using linguistic features, however, benefit substantially, with accuracy leveling off after 150,000 training cases. With only 2,000 training cases, the classifiers built using all features range in accuracy from $75.06 \%$ to $78.84 \%$, substantially above the baseline accuracy of $50 \%$.

\section{Discussion}

As the results in section 4 show, it is possible to build classifiers that can distinguish human reference translations from the output of a machine translation system with high accuracy. We thus have an automatic mechanism that can perform the task that humans appear to do with ease, as noted in section 1 . The best result, a classifier with $82.89 \%$ accuracy, is achieved by combining perplexity calculations with a set of finer-grained linguistic features. Even with as few as 2,000 training cases, accuracy exceeded $75 \%$. In the discussion below we consider the advantages and possible uses of this automatic evaluation methodology. 


\subsection{Advantages of the approach}

Once an appropriate set of features has been selected and tools to automatically extract those features are in place, classifiers can be built and evaluated quickly. This overcomes the two problems associated with traditional manual evaluation of MT systems: manual evaluation is both costly and time-consuming. Indeed, an automated approach is essential when dealing with an MT system that is under constant development in a collaborative research environment. The output of such a system may change from day to day, requiring frequent feedback to monitor progress.

The methodology does not crucially rely on any particular set of features. As an MT system matures, more and more subtle cues might be necessary to distinguish between human and machine translations. Any linguistic feature that can be reliably extracted can be proposed as a candidate feature to the decision tree tools.

The methodology is also not sensitive to the domain of the training texts. All that is needed to build classifiers for a new domain is a sufficient quantity of aligned translations.

\subsection{Possible applications of the approach}

The classifiers can be used for evaluating a system overall, providing feedback to aid in system development, and in evaluating individual sentences.

\section{Evaluating an MT system overall}

Evaluating the accuracy of the classifier against held-out data is equivalent to evaluating the fluency of the MT system. As the MT system improves, its output will become more like the human reference translations. To measure improvement over time, we would hold the set of features constant and build and evaluate new classifiers using the human reference translations and the output of the MT system at a given point in time. Using the same set of features, we expect the accuracy of the classifiers to go down over time as the MT output becomes more like human translations.

\section{Feedback to aid system development}

Our primary interest in evaluating an MT system is to identify areas that require improvement. This has been the motivation for using linguistic features in addition to perplexity measures. From the point of view of system development, perplexity is a rather opaque measure. This can be viewed as both a strength and a weakness. On the one hand, it is difficult to tune a system with the express goal of causing perplexity to improve, rendering perplexity a particularly good objective measurement. On the other hand, given a poor perplexity score, it is not clear how to improve a system without additional failure analysis.

We used the DNETVIEWER tool (Heckerman et al., 2000), a visualization tool for viewing decision trees and Bayesian networks, to explore the decision trees and identify problem areas in our MT system. In one visualization, shown in Figure 2, DNETVIEWER allows the user to adjust a slider to see the order in which the features were selected during the heuristic search that guides the construction of decision trees. The most discriminatory features are those which cause the MT translations to look most awful, or are characteristics of the reference translations that ought to be emulated by the MT system. For the coarse model shown in Figure 2, the distance between pronouns (nPronDist) is the highest predictor, followed by the number of second person pronouns (n2ndPersPron), the number of function words (nFunctionWords), and the distance between prepositions (nPrepDist).

Using DNETVIEWER we are able to explore the decision tree, as shown in Figure 3. Viewing the leaf nodes in the decision tree, we see a probability distribution over the possible states of the target variable. In the case of the binary classifier here, this is the probability that a sentence will be a reference translation. In Figure 3, the topmost leaf node shows that $\mathrm{p}$ (Human translation) is low. We modified DNETVIEWER so that double-clicking on the leaf node would display reference translations and MT sentences from the training data. We display a window showing the path through the decision tree, the probability that the sentence is a reference translation given that path, and the sentences from the training data identified by the features on the path. This visualization allows 
the researcher to view manageable groups of similar problem sentences with a view to identifying classes of problems within the groups. A goal for future research is to select additional linguistic features that will allow us to pinpoint problem areas in the MT system and thereby further automate failure analysis.

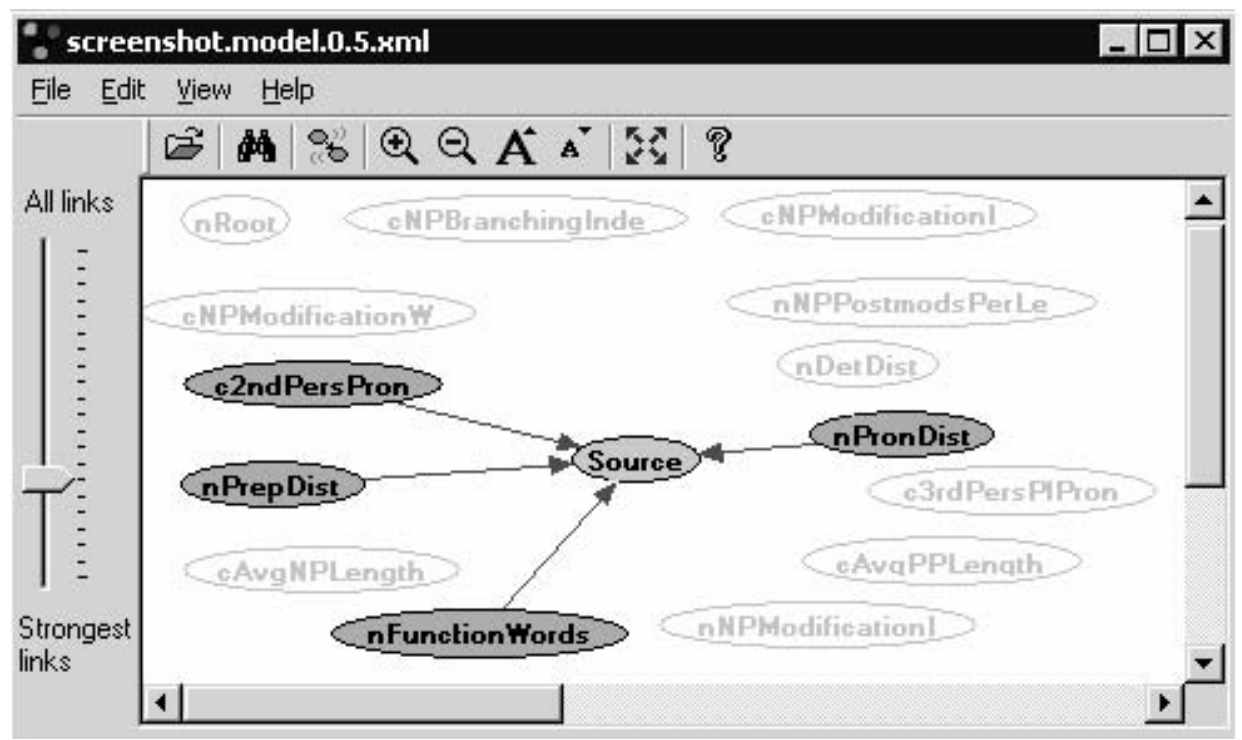

Figure 2 Using the slider to view the best predictors

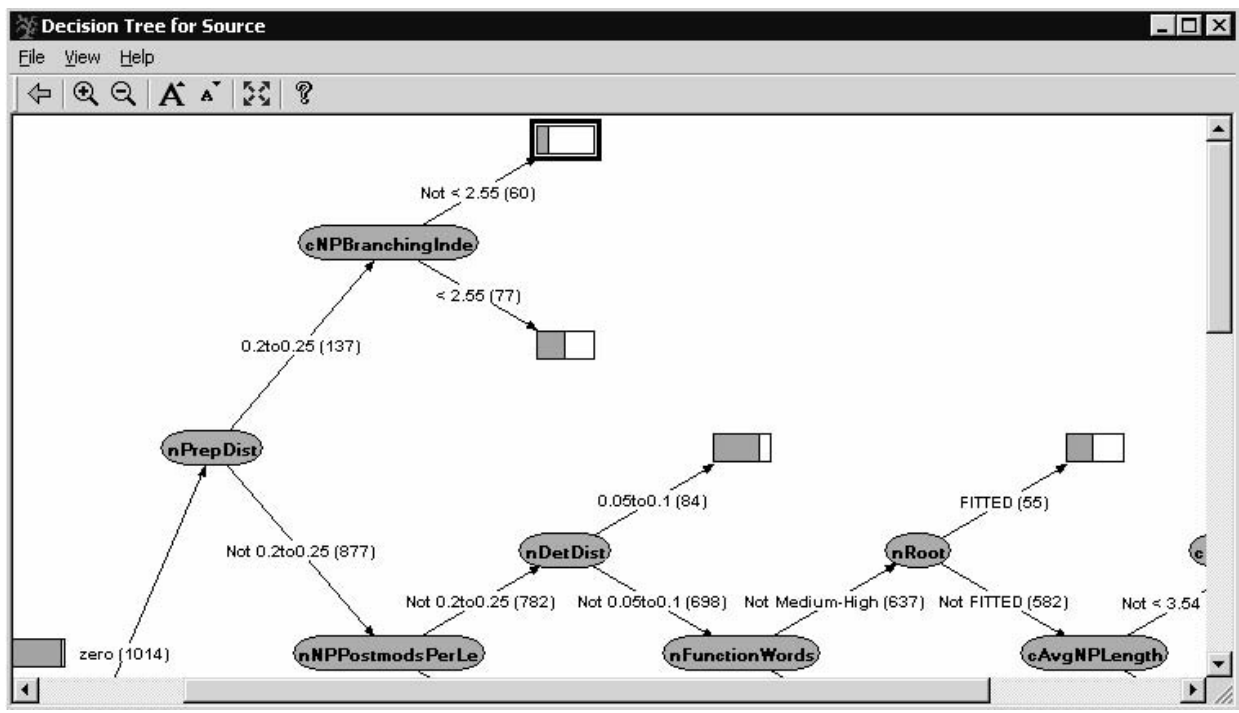

Figure 3 Examining sentences at a leaf node in the decision tree 


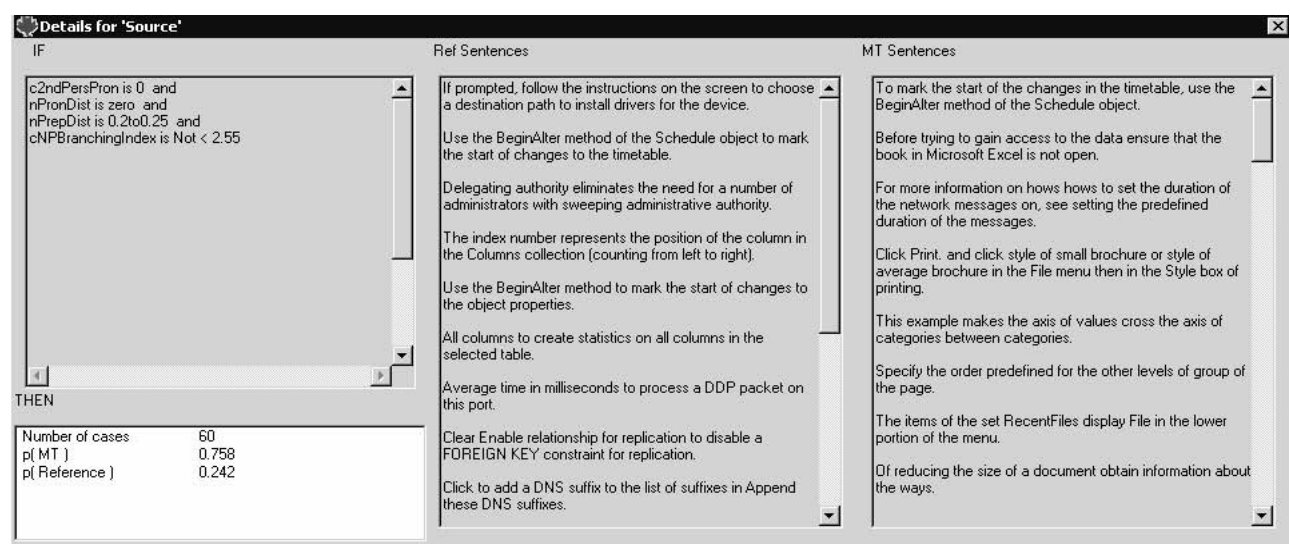

Figure 4 Examining sentences at a leaf node in the decision tree

Decision trees are merely one form of classifier that could be used for the automated evaluation of an MT system. In preliminary experiments, the accuracy of classifiers using support vector machines (SVMs) (Vapnik, 1998; Platt et al., 2000) exceeded the accuracy of the decision tree classifiers by a little less than one percentage point using a linear kernel function, and by a slightly greater margin using a polynomial kernel function of degree three. We prefer the decision tree classifiers because they allow a researcher to explore the classification system and focus on problem areas and sentences. We find this method for exploring the data more intuitive than attempting to visualize the location of sentences in the highdimensional space of the corresponding SVM.

\section{Evaluating individual sentences}

In addition to system evaluation and failure analysis, classifiers could be used on a persentence basis to guide the output of an MT system by selecting among multiple candidate strings. If no candidate is judged sufficiently similar to a human reference translation, the sentence could be flagged for human postediting.

\section{Conclusion}

We have presented a method for evaluating the fluency of MT, using classifiers based on linguistic features to emulate the human ability to distinguish MT from human translation. The techniques we have described are system- and language-independent. Possible applications of our approach include system evaluation, failure analysis to guide system development, and selection among alternative possible outputs.

We have focused on structural aspects of a text that can be used to evaluate fluency. A full evaluation of MT quality would of course need to include measurements of idiomaticity and techniques to verify that the semantic and pragmatic content of the source language had been successfully transferred to the target language.

\section{Acknowledgements}

Our thanks go to Eric Ringger and Max Chickering for programming assistance with the tools used in building and evaluating the decision trees, and to Mike Carlson for help in sampling the initial datasets. Thanks also to John Platt for helpful discussion on parameter setting for the SVM tools, and to the members of the MSR NLP group for feedback on the uses of the methodology presented here.

\section{References}

Alshawi, H., S. Bangalore, and S. Douglas. 1998. Automatic acquisition of hierarchical transduction models for machine translation. In Proceedings of the $36^{\text {th }}$ Annual Meeting of the Association for Computational Linguistics, Montreal Canada, Vol. I: 41-47.

Bangalore, S., O. Rambow, and S. Whittaker. 2000. Evaluation Metrics for Generation. In Proceedings of the International Conference on Natural Language Generation (INLG 2000), Mitzpe Ramon, Israel. 1-13.

Chickering, D. M., D. Heckerman, and C. Meek. 1997. A Bayesian approach to learning Bayesian 
networks with local structure. In Geiger, D. and P. Punadlik Shenoy (Eds.), Uncertainty in Artificial Intelligence: Proceedings of the Thirteenth Conference. 80-89.

Clarkson, P. and R. Rosenfeld. 1997. Statistical Language Modeling Using the CMU-Cambridge Toolkit. Proceedings of Eurospeech97. 27072710.

Heckerman, D., D. M. Chickering, C. Meek, R. Rounthwaite, and C. Kadie. 2000. Dependency networks for inference, collaborative filtering and data visualization. Journal of Machine Learning Research 1:49-75.

Heidorn, G. E., 2000. Intelligent writing assistance. In R. Dale, H. Moisl and H. Somers (Eds.). Handbook of Natural Language Processing. New York, NY. Marcel Dekker. 181-207.

Langkilde, I., and K. Knight. 1998. Generation that exploits corpus-based statistical knowledge. In Proceedings of the $36^{\text {th }}$ Annual Meeting of the Association for Computational Linguistics, and $17^{\text {th }}$ International Conference on Computational Linguistics, Montreal, Canada. 704-710.

Nyberg, E. H., T. Mitamura, and J. G. Carbonnell. 1994. Evaluation Metrics for Knowledge-Based Machine Translation. In Proceedings of the $15^{\text {th }}$ International Conference on Computational Linguistics, Kyoto, Japan (Coling 94). 95-99.

Platt, J., N. Cristianini, J. Shawe-Taylor. 2000. Large margin DAGs for multiclass classification. In Advances in Neural Information Processing Systems 12, MIT Press. 547-553.

Richardson, S., B. Dolan, A. Menezes, and J. Pinkham. 2001. Achieving commercial-quality translation with example-based methods. Submitted for review.

Ringger, E., M. Corston-Oliver, and R. Moore. 2001. Using Word-Perplexity for Automatic Evaluation of Machine Translation. Manuscript.

Su, K., M. Wu, and J. Chang. 1992. A new quantitative quality measure for machine translation systems. In Proceedings of COLING92, Nantes, France. 433-439.

Vapnik, V. 1998. Statistical Learning Theory, WileyInterscience, New York. 